\title{
USO DA ULTRA-SONOGRAFIA NA TRIAGEM DE DISRAFISMOS ESPINHAIS OCULTOS
}

\author{
José Gilberto de Brito Henriques', Geraldo Pianetti Filho², Petrônio Rabelo Costa³, \\ Karina Santos Wandeck Henriques ${ }^{4}$, Francisco Otaviano Lima Perpétuo ${ }^{5}$
}

\begin{abstract}
RESUMO - O uso da ultra-sonografia para diagnóstico de lesões da coluna vertebral é difundido desde a década de oitenta. Suas aplicações envolvem: diagnóstico de lesões medulares traumáticas; uso intra-operatório em cirurgias do trauma e tumores medulares; uso pré-operatório e evolutivo em pacientes com disrafismos espinhais; diagnóstico pré-natal dos disrafismos; diagnóstico dos disrafismos espinhais ocultos ao nascimento. Pacientes com suspeita de disrafismo espinhal oculto (estigmas cutâneos em linha média ou história familiar de disrafismos) representaram a principal indicação do exame que foi realizado em 292 pacientes do Hospital das Clínicas da Universidade Federal de Minas Gerais. Alterações como lipomas do cone medular, medula baixa, filo terminal espesso, cistos da ponta do cone medular e seios dérmicos foram identificados com fidelidade à ultra-sonografia. A interpretação do exame não apresentou dificuldades técnicas. Sua realização é rápida, não é necessária sedação, apresenta baixo custo e idealmente deve ser realizado pelo próprio neurocirurgião devido ao grande número de informações possíveis durante o exame. 0 exame entretanto não substitui a ressonância magnética mas pode ser um bom método de triagem para os pacientes.
\end{abstract}

PALAVRAS-CHAVE: disrafismo espinhal oculto, ultra-sonografia, diagnóstico.

\begin{abstract}
Screening of occult spinal dysraphism by ultrasonography
ABSTRACT - Ultrasonography for diagnosis of spinal cord diseases has been used since the eighties. There are different fields for its use: traumatic spine lesions, intra-operative use in trauma and spine tumors surgery, pre-operative and follow up study for spinal dysraphism, occult spinal dysraphism of the newborn. Patients with suspected occult spinal dysraphism are the most important indication for the method. Utrasonography was performed in 292 patients of Hospital das Clínicas, Universidade Federal de Minas Gerais. Lesions such as conus medullaris lipomas, low level of conus medullaris, filum terminale tethering, cauda equina cysts and dermal sinuses can be well demonstrated. There is no difficulty in interpreting the image. The exam does not need sedation; it is safe, fast and cheap. Ideally it should be performed by the neurosurgeon as it may provide many information during the examination. The ultrasonography does not replace magnetic resonance imaging but it is an excellent method for screening.
\end{abstract}

KEY WORDS: occult spinal dysraphism, ultrasound, diagnosis.

O uso da ultra-sonografia (US) para diagnóstico de lesões da coluna vertebral é difundido desde a década de oitenta do século passado. Suas aplicações envolvem: diagnóstico de lesões medulares traumáticas ${ }^{1,2}$; uso intra-operatório em cirurgias do trauma e tumores medulares ${ }^{3-5}$; uso pré-operatório e evolutivo em pacientes com disrafismos espinhais ${ }^{6,7}$; diagnóstico pré-natal dos disrafismos ${ }^{8}$; diagnóstico dos disrafismos espinhais ocultos ao nascimento.

Em neurocirurgia pediátrica, a US de coluna é facilitada, principalmente em recém-nascidos, devi- do à ossificação incompleta dos elementos posteriores das vértebras mais caudais, o que permite a visibilização do conteúdo do canal vertebral e estruturas ósseas. Apresenta boa correspondência com achados de ressonância magnética ${ }^{9}(\mathrm{RM})$. $O$ exame é de fácil e rápida realização, inócuo ${ }^{10}$, pode ser feito com o paciente em seu leito, permite o diagnóstico precoce e a triagem de pacientes com lesões no neuro-eixo.

Pacientes com suspeita de disrafismo espinhal oculto (estigmas cutâneos em linha média ou histó-

Hospital das Clínicas (HC) e Faculdade de Medicina (FM) da Universidade Federal de Minas Gerais (UFMG), Belo Horizonte MG, Brasil: ${ }^{1}$ Médico Residente de Neurocirurgia do HC UFMG; ${ }^{2}$ Professor do Departamento de Neurologia da FM UFMG, Coordenador da Neurocirurgia Pediátrica do HC UFMG; ${ }^{3}$ Professor do Departamento de Pediatria da FM UFMG, Preceptor da Residência de Radiologia do HC UFMG; ${ }^{4}$ Médica Residente de Pediatria do HC UFMG; ${ }^{5}$ Professor do Departamento de Neurologia da FM UFMG, Neurorradiologista, Preceptor da Residência de Neurocirurgia do HC UFMG.

Recebido 7 Agosto 2003, recebido na forma final 14 Janeiro 2004. Aceito 2 Março 2004.

Dr. José Gilberto de Brito Henriques - Avenida Augusto de Lima 196/902 - 30190-001 Belo Horizonte MG - Brasil. E-mail: henriques_jgb@hotmail.com 
ria familiar de disrafismos) constituem a principal indicação da US. A US não substitui a RM mas pode triar os pacientes que realmente necessitam do exame.

Quanto mais novo o paciente, melhor a qualidade da imagem, devido à janela conseguida pela ossificação parcial dos elementos posteriores das vértebras. A partir dos nove meses de idade, o padrão do exame já pode ser muito precário para se definir a conduta.

Apresentamos nossa experiência com a US na triagem de disrafismos espinhais.

\section{MÉTODO}

No Serviço de Neurocirurgia do Hospital das Clínicas da UFMG foram realizadas US de coluna vertebral em 292 pacientes; dentre eles, 144 apresentavam estigmas cutâneos em linha média. O paciente é colocado em decúbito ventral com coxim sob o tórax e abdome, o que permite a flexão da coxa. É solicitado ao acompanhante que alimente a criança minutos antes do exame ou mesmo no decorrer deste, para se obter melhor cooperação do paciente sem necessidade de sedação. $O$ decúbito lateral pode ser usado quando é necessário avaliar a mobilidade das raízes da cauda eqüina.

É utilizada sonda linear de 5-9 MHz. e gel para US aquecido.

\section{RESULTADOS}

Alterações da ultra-sonografia - Dos 144 pacientes com estigma cutâneo em linha média, 14 apresentaram alterações à US: 4 seios dérmicos; um lipoma intra-raqueano; dois disrafismos ósseos acima de L3; um cone medular abaixo de L3 e 6 cistos de cone medular.

Anatomia normal - A medula espinhal apresenta-se hipo-ecogênica, levemente mais ecogênica que o líquor que a cerca, sendo sua superfície bem delimitada em todo o seu trajeto (Fig 1). Em sua parte central, no corte sagital, a medula possui uma ou duas linhas ecogênicas que são denominadas "complexo ecóico central" cuja interpretação é ainda discutida: se representa o canal central da medula ou a interface entre a superfície e a profundidade da comissura anterior ${ }^{11}$ (Fig 1). A medula apresenta diferentes formas em seus segmentos. A medula cervical apresenta forma oval e espessada no corte axial (devido à intumescência cervical); já o segmento torácico é mais estreito e arredondado. Em determinados níveis torácicos é possível visibilizar os ligamentos denteados que fixam a medula na porção central do canal vertebral. A transição tóraco-lombar apresenta-se arredondada e mais espessa que o segmento torácico devido à intumescência lombar.

No recém-nascido, o cone medular encontra-se geralmente no nível das vértebras L1-L2 variando de T11 a L $3^{12,13}$. A localização do cone medular pode ser determinada através da palpação de pontos referenciais: o ápice da décima segunda costela que corresponde à vértebra $L 2$; e a crista ilíaca que indica L4-L5. Entretanto, ambos os referenciais podem apresentar erros. O nível deve ser confirmado através da contagem das vértebras com a US ou marcando-se a pele com material radiopaco no nível do cone medular para posterior comparação com radiografia da coluna. Quando o cone medular atinge o corpo de L3 considera-se um nível baixo.

A cauda eqüina é reconhecida como um emaranhado de linhas ecogênicas que saem da superfície ventral e dorsal do cone medular e tendem a assumir a posição mais ventral (Fig 2). Por vezes, é possível evidenciar pequenas raízes individualizadas, saindo mais proximalmente do canal vertebral. No plano axial, as raízes envolvem a medula por todos os lados (Fig 3).

O exame de US da medula é dinâmico. Tanto o cone medular quanto as raízes nervosas movimentam-se de acordo com a respiração e o ciclo cardíaco; movimentam-se também em direção cranial com a flexão do pescoço. A perda destes movimentos pode ser sinal importante que acompanha os casos de medula presa.

O filo terminal (Fig 2) apresenta-se com sinal mais ecogênico e mais espesso que as raízes nervosas. Por vezes pode apresentar sinal duplo (duas linhas hiperecogênicas). Inicia-se no ápice do cone medular e, diferentemente das raízes nervosas que tendem a adotar direção ventral, coloca-se mais posteriormente, em direção ao fundo do saco dural, a partir de onde passa a ser denominado ligamento terminal, que se insere no hiato sacral. O filo terminal também pulsa como o cone medular e as raízes.

A dura-máter é facilmente identificada nos cortes sagitais e axiais como uma faixa hiperecogênica que delimita todo o canal vertebral (Figs 1, 2 e 3). Em seu segmento final, o saco dural é mais difícil de ser identificado, devido à convergência das raízes nervosas, ao estreitamento do espaço que ocorre no fechamento do saco dural (aproximadamente em S2) e à cifose fisiológica sacro- coccígea (Fig 4).

As vértebras não são bem evidenciadas à US. É possível evidenciar os processos espinhosos e os corpos vertebrais como estruturas hiperecogênicas, po- 
rém sem detalhes que permitam avaliá-las adequadamente (Figs 1 e 2). As vértebras coccígeas não são calcificadas no recém-nascido, o que resulta numa imagem hipo-ecogênica que pode causar erros na interpretação do exame, principalmente nos cortes axiais, onde podem ser confundidas com o saco dural.

Aplicação clínica da ultra-sonografia - Alterações no desenvolvimento do sistema nervoso central, que ocorrem devido a falhas no fechamento do tubo neural durante a quarta semana da embriogênese, resultam nos disrafismos. A incidência destes defeitos mostra variação geográfica significativa ( 0,5 a 5 casos a cada 1000 nascimentos) e acometem menos freqüentemente os negros. Sua prevalência é maior em classes sociais menos favorecidas ${ }^{14-16}$.

Com base na forma de apresentação clínica, os disrafismos espinhais podem ser divididos em abertos, fechados e ocultos(DO). Os DO podem não apresentar manifestações clínicas no recém-nascido mas podem vir associados a estigmas cutâneos que indicam o disrafismo: lipomas, tufo de pêlos, fossetas sacro-coccígeas (dimples), alterações no sulco glúteo, apêndices cutâneos, dentre outras menos comuns É na abordagem inicial destes pacientes que a US tem fundamental importância.

O diagnóstico dos disrafismos pode ser feito no período pré-natal, ao nascimento e tardiamente (nos estirões de crescimento). O diagnóstico prénatal com a US tem sensibilidade de $75 \%{ }^{17}$ e permite o acompanhamento da lesão durante a gestação assim como o planejamento obstétrico (indicação de parto cirúrgico). O diagnóstico ao nascimento é realizado com o exame físico. Já o diagnóstico tardio é feito nos períodos de estirão, ou mesmo na vida adulta quando ocorrem alterações motoras nos membros inferiores, distúrbios esfincterianos, disfunção erétil, dor lombar. Este diagnóstico tardio pode ser evitado em pacientes com estigmas cutâneos em linha mediana se forem investigados com a US. A radiografia simples da coluna vertebral tem valor limitado em recém-nascidos, pois pode mostrar o fechamento incompleto dos arcos vertebrais mais inferiores, o que não significa necessariamente disrafismo espinhal ${ }^{18}$, além de expor o recém-nascido à radiação.

\section{DISCUSSÃo}

A medula presa ocorre devido a fixação patológica do cone medular, que causa tração da medula resultando em distorção da anatomia normal e em lesões isquêmicas que ocorrem com o crescimento da criança e com as atividades diárias. Diversas alterações podem ser vistas à US: o cone medular atinge L3 ou está abaixo dele (Fig 5); diminuição da pulsação fisiológica da medula e raízes nervosas; posição excêntrica no canal vertebral (o cone tende a ficar lateralizado e desviado para a parte dorsal do canal).

O seio dérmico é pequena malformação que se apresenta como uma depressão ("dimple") e um canal fistuloso que liga o saco dural à parte externa da pele. A lesão pode terminar em fundo cego (dimple raso) quando não há comunicação com o saco dural. A US nos pacientes com seio dérmico mostra estreito trajeto hipo-ecogênico que se inicia na pele e chega até o saco dural (Fig 6). Na maioria das vezes não é possível, à US, evidenciar a comunicação entre o seio dérmico e a dura-máter. $O$ exame permite apenas inferir a possibilidade da comunicação. A RM pode confirmar o diagnóstico.

O lipoma intra-raqueano apresenta-se como lesão tumoral na linha mediana ou paramediana, de consistência macia, aderida aos planos profundos. A lipomielomeningocele é um disrafismo espinhal em que o lipoma invade o saco dural, podendo envolver as raízes nervosas e o cone medular. Há indicação cirúrgica na maioria dos casos porque as manifestações neurológicas, quando não estão presentes já ao nascimento, ocorrem com o crescimento do paciente, devido ao ancoramento da medula. A US pode mostrar a invasão do canal vertebral e o envolvimento do cone medular (Figs 7 e 8). A tomografia computadorizada (TC) e a RM são exames necessários para confirmar a US e permitir o planejamento cirúrgico.

O diagnóstico diferencial destas lesões também é facilitado com a US. O paciente da Figura 9 nasceu com lesão dorsal sugestiva de lipoma. A US mostrou que se tratava de provável linfangioma e permitiu excluir a comunicação da lesão com o canal vertebral.

A diastematomielia é divisão parcial ou completa da medula ou cone medular, em forma de fenda, que ocorre devido a um septo ósseo, fibroso ou cartilaginoso que se encontra entre as duas "hemimedulas", fixando-a em um determinado nível. Com o crescimento do paciente e a ascensão da medula, este septo vai dividindo a medula em duas partes. A US pode evidenciar o ancoramento da medula e, ao corte axial, a sua divisão. 


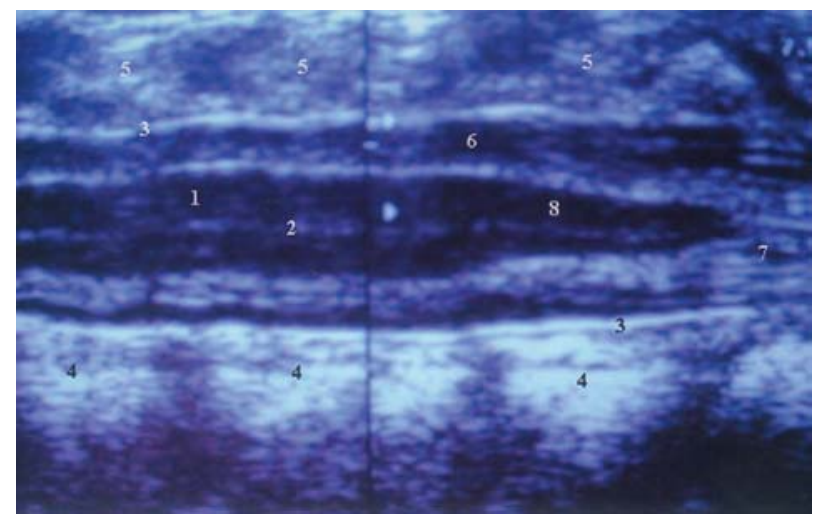

Fig 1. Ultra-sonografia sagital lombar: 1) medula espinhal; 2) complexo ecóico central; 3) dura-máter; 4) corpo vertebral; 5) processo espinhoso; 6) espaço subaracnóideo; 7) raízes nervosas; 8) cone medular.

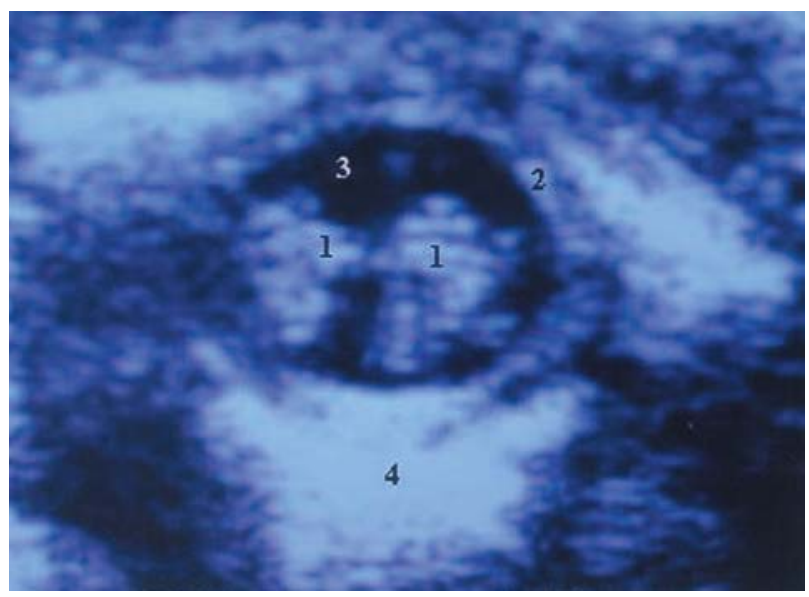

Fig 3. Ultra-sonografia axial lombar. Cauda eqüina: 1) raízes nervosas; 2) dura-máter; 3) espaço subaracnóideo; 4) corpo vertebral.

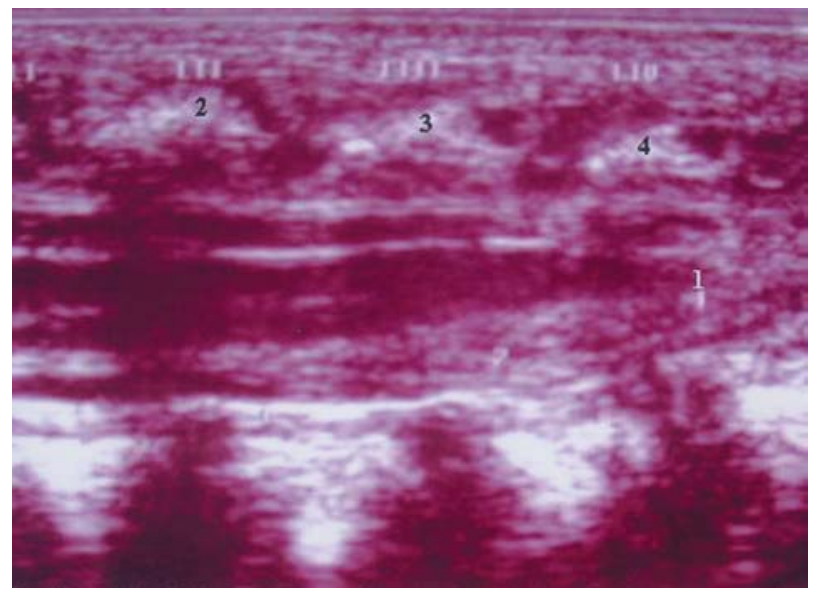

Fig 5. Ultra-sonografia sagital lombar. Medula baixa: 1) ápice do cone medular; 2) processo espinhoso L2; 3) processo espinhoso L3; 4) processo espinhoso $L 4$.

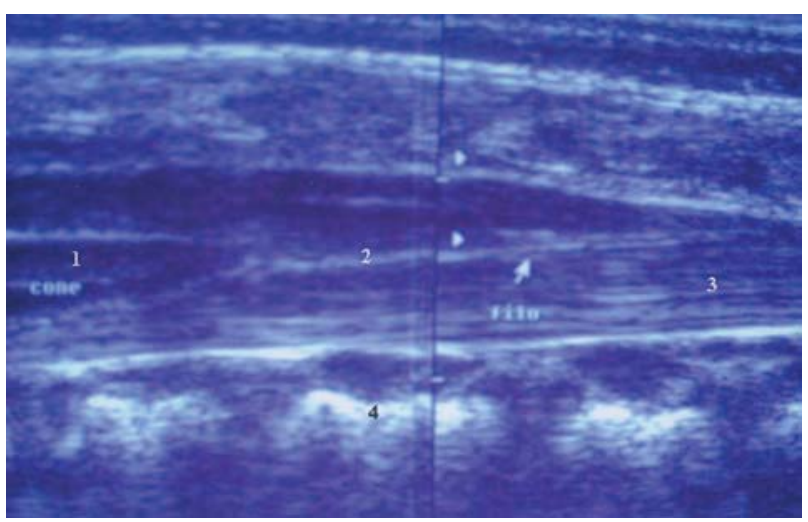

Fig 2. Ultra-sonografia sagital lombar: 1) cone medular; 2) filo terminal; 3) cauda eqüina; 4) corpo vertebral.

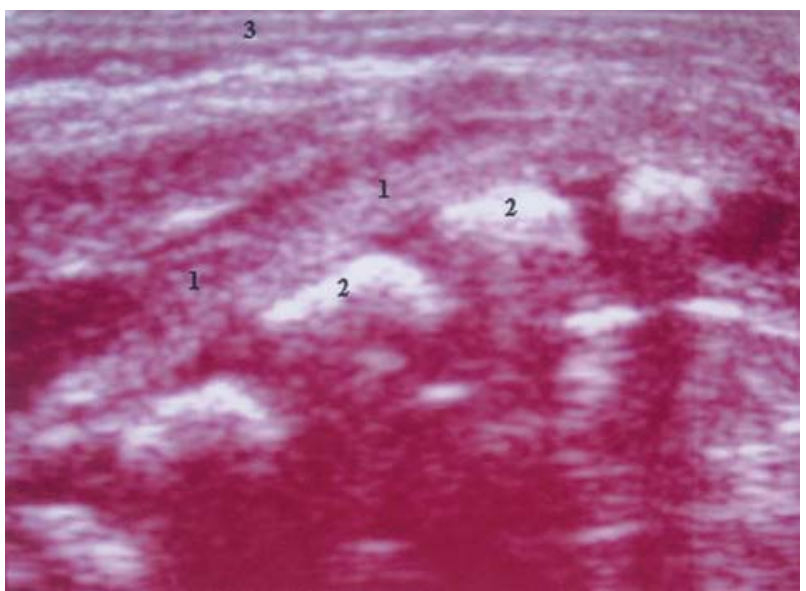

Fig 4. Ultra-sonografia sagital sacral: 1) cauda equina; 2) corpo vertebral; 3) subcutâneo.

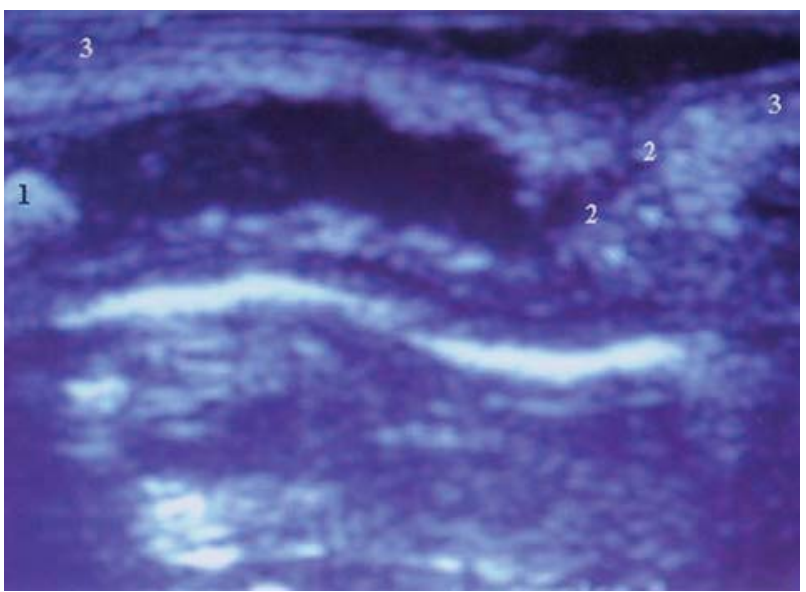

Fig 6. Ultra-sonografia sagital. Seio dérmico: 1) vértebra sacral; 2) seio dérmico; 3) subcutâneo. 


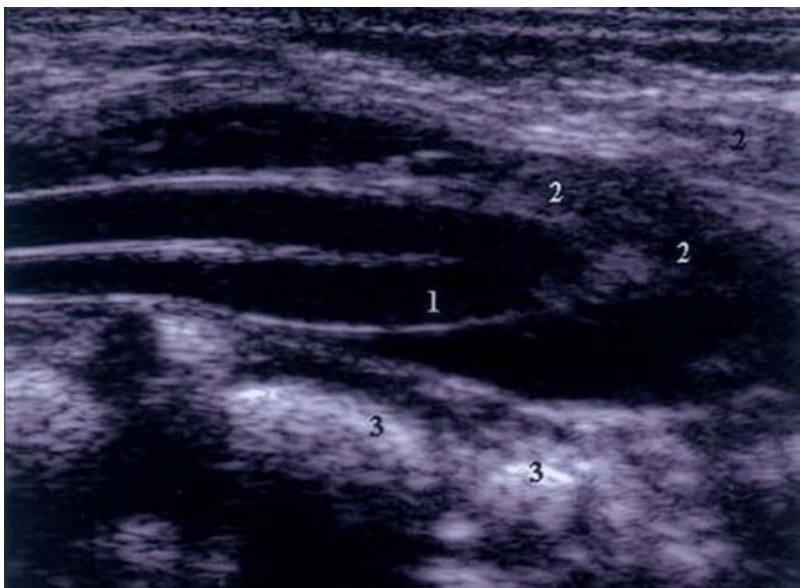

Fig 7. Ultra-sonografia sagital lombar. Lipoma; 1) cone medular (com a anatomia distorcida); 2) lipoma invadindo canal vertebral e envolvendo cone medular; 3) corpos vertebrais malformados.

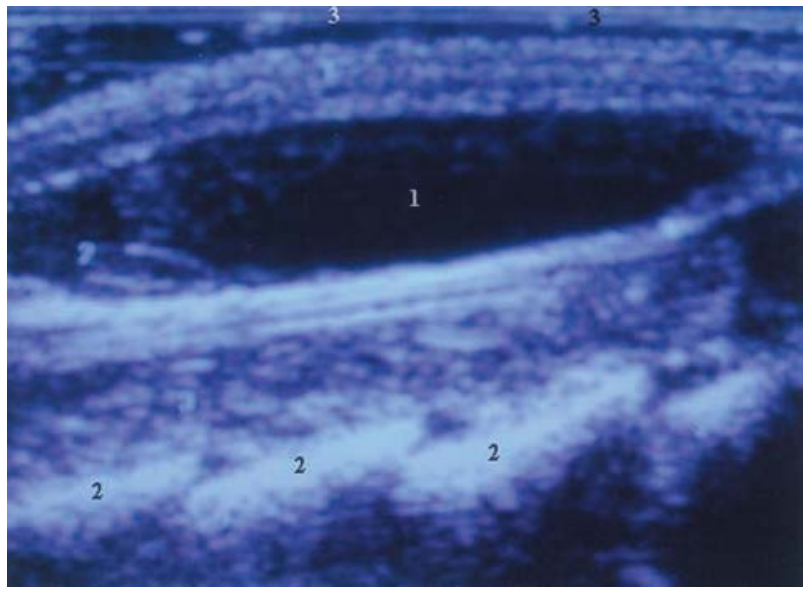

Fig 9. Ultra-sonografia sagital. Linfangioma: 1) lesão cística bem delimitada (linfangioma); 2) processos espinhosos vertebrais (não há invasão do canal vertebral); 3) Subcutâneo.

Pequenos cistos podem ser vistos na extremidade do cone medular e entre as raízes da cauda eqüina (Fig 10). Geralmente são cistos aracnóides sem importância clínica. Existem também os chamados cistos filares, que são remanescentes de células embrionárias mais caudais, que se acumulam no filo terminal e que tenderiam a formar um "ventrículo terminal" ou "quinto ventrículo"11. Estes cistos são geralmente pequenos e encontrados incidentalmente em crianças saudáveis. Entretanto eles podem acompanhar a medula presa e a mielomeningocele.

Em conclusão, a US da medula e coluna vertebral tem diversas aplicações. Como método de triagem para pacientes com suspeita de disrafismo espinhal oculto é extremamente útil e ainda pouco utilizada em nosso meio. Seu uso nos pacientes com disrafis-

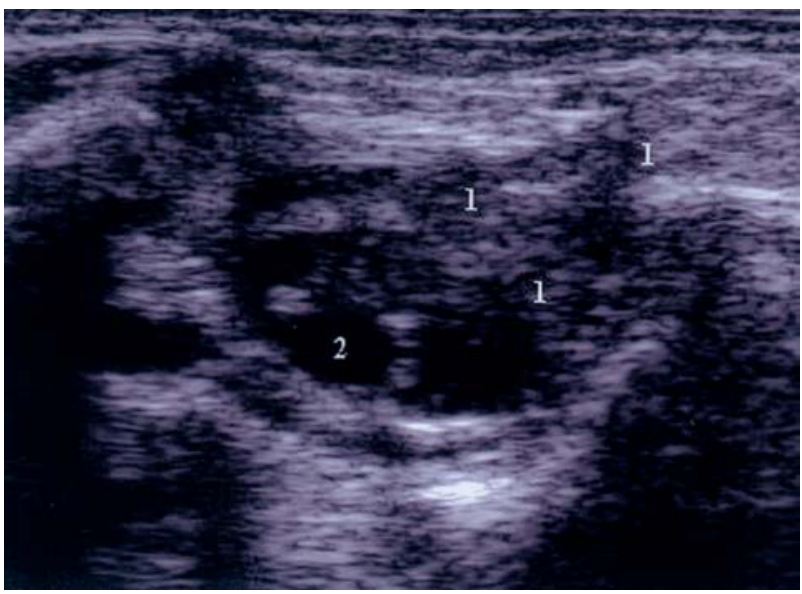

Fig 8. Ultra-sonografia axial lombar. Lipoma: 1) lipoma invadindo o canal vertebral; 2) espaço subaracnóideo.

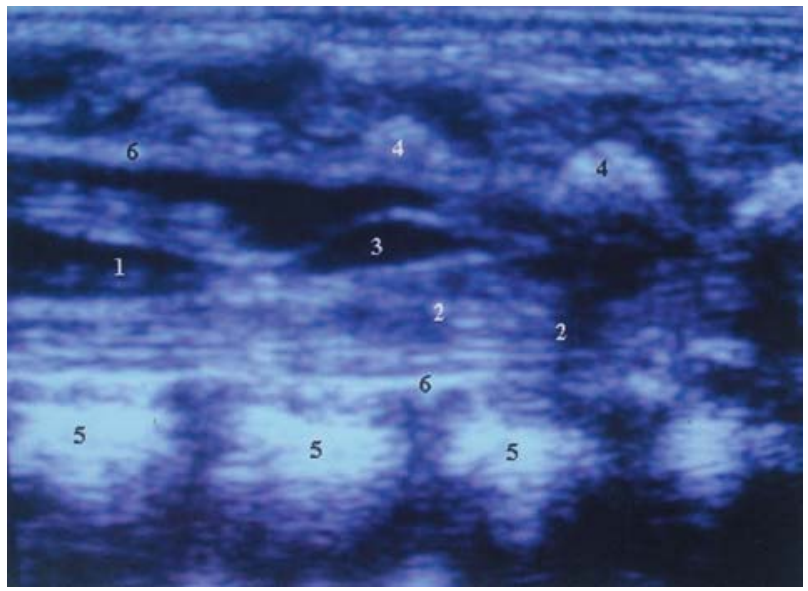

Fig 10. Ultra-sonografia sagital lombar. Cisto: 1) cone medular; 2) raízes nervosas; 3) cisto; 4) processo espinhoso; 5) corpo vertebral; 6) dura-máter.

mo aberto ou fechado também apresenta benefícios, porém o diagnóstico destas doenças é feito com a ectoscopia e o exame não substitui ou dispensa a TC e/ou RM.

A interpretação do exame não apresenta dificuldades técnicas. $O$ exame é rápido, não necessita de sedação, apresenta baixo custo e idealmente deve ser realizado pelo próprio neurocirurgião dado o grande número de informações possíveis durante sua realização. Deve-se ter em mente, entretanto, que a US de coluna vertebral é exame de triagem. Na maioria das vezes, quando a US mostrar algum tipo de alteração, a RM será o melhor método de imagem para confirmação do diagnóstico.

Agradecimentos - Aos Professores Hélio Rubens Machado, Sebastião Gusmão, Eduardo Carlos Tavares, Hen- 
rique Vitor Leite; à Dra Márcia Cristina da Silva; aos Drs. Tales Henrique Ulhôa, Alex Nagem Machado; e ao acadêmico Teófilo Pires.

\section{REFERÊNCIAS}

1. Filippigh P, Clapuyt P, Debauche C, Claus D. Sonographic evaluation of traumatic spinal cord lesions in the newborn infant. Pediatr Radiol 1994;24:245-247.

2. Montalvo BM, Quencer RM, Green BA, Eismont FJ, Brown MJ, Brost P. Intraoperative sonography in spinal trauma. Radiology 1984;153:125134.

3. Mirvis SE, Geisler FH. Intraoperative sonography of cervical spinal cord injury: results in 30 patients. AJNR Am J Neuroradiol 1990;11:755-761.

4. Rubin JM, DiPietro MA, Chandler WF, Venes JL. Spinal ultrasonography: intraoperative and pediatric applications. Radiol Clin N Am 1988;26:127.

5. Maiuri F, Iaconetta G, Gallicchio B, Stella L. Intraoperative sonography for spinal tumors correlations with MR findings and surgery. J Neurosurg Sci 2000;44:115-122.

6. Byrd SE, Radkowski MA. The radiological evaluation of the child with a myelomeningocele. J Natl Med Assoc 1991;83:608-614.

7. Coniglio SJ, Anderson SM, Ferguson JE 2nd. Functional motor outcome in children with myelomeningocele: correlation with anatomic level on prenatal ultrasound. Dev Med Child Neurol 1996;38:675-680.

8. Sattar TS, Bannister CM, Russell SA, Rimmer S. Pre-natal diagnosis of occult spinal dysraphism by ultrasonography and post-natal evaluation by MR scanning. Eur J Pediatr Surg 1998;8(Suppl):31-33.

9. Korsvik HE, Keller MS. Sonography of occult dysraphism in neonates and infants with MR imaging correlation. Radiographics 1992;12:297-308.

10. Barr LL. Clinical concerns in the ultrasound exposure of the developing central nervous system. Ultrasound Med Biol 2001;27:889-892.

11. DiPietro MA. The pediatric spinal canal. In Rumack CM, Wilson S, Charboneau JW (eds). Diagnostic ultrasound. New York: Mosby, 1998:1589-1615.

12. Hill CA, Gibson PJ. Ultrasound determination of the normal location of the conus medullaris in neonates. Am J Neuroradiol 1995;16:469-472.

13. DiPietro MA. The conus medullaris: normal US findings throughout childhood. Radiology 1993;188:149-153.

14. Pianetti G, Herval LMA, Henriques JGB. Espinha bífida. In Fonseca LF, Pianetti G, Xavier CC (eds). Compêndio de neurologia infantil. Belo Horizonte: Medsi, 2002:211-215.

15. Northrup H, Volcik KA. Spina bifida and other neural tube defects. Curr Probl Pediatr 2000;30:313-332.

16. Cornette L, Verpoorten C, Lagae L, et al. Closed spinal dysraphism: a review on diagnosis and treatment in infancy. Eur J Pediatr Neurol 1998;2:179-85.

17. Boyd PA, Wellesley, De Walle HE, et al. Evaluation of the prenatal diagnosis of neural tube defects by fetal ultrasonographic examination in different centres across Eur J Med Screen 2000;7:169-174.

18. Silverman FN, Kuhn JP. Coffey's pediatric X-ray diagnosis: an integrated imaging approach. 9.Ed. New York: Mosby, 1993;621-623. 